Einführung zum Thema

Onkologe $2019 \cdot 25: 556-558$

https://doi.org/10.1007/s00761-019-0596-z

Online publiziert:29. Mai 2019

(c) Springer Medizin Verlag GmbH, ein Teil von

Springer Nature 2019

\section{Christine Spitzweg' ${ }^{1}$ P. M. Schlag}

${ }^{1}$ Medizinische Klinik und Poliklinik IV, Interdisziplinäres Schilddrüsenzentrum am Klinikum der Universität München (ISKUM), Klinikum der Universität München, LMU München, München, Deutschland

${ }^{2}$ Charite Comprehensive Cancer Center, Berlin, Deutschland

\title{
Erfolgreiche Weiterentwicklung multidisziplinärer Diagnostik und Therapie bei malignen Schilddrüsentumoren
}

Auf dem Gebiet der malignen Schilddrüsentumoren haben sich insbesondere durch eine zunehmende und verfeinerte molekulargenetische Charakterisierung zahlreiche neue diagnostische und therapeutische Entwicklungen ergeben.

\section{) Ein gezielteres, risikoadap- tiertes und individualisiertes Vorgehen wird angestrebt}

Hierdurch kann ein zunehmend gezielteres, risikoadaptiertes und individualisiertes Vorgehen erreicht werden (Precision Medicine). Das vorliegende Themenheft resümiert für alle Entitäten des Schilddrüsenkarzinoms die aktuellste Datenlage im interdisziplinären Kontext unter Berücksichtigung aktueller internationaler Leitlinien.

Im ersten Beitrag geben Führer, Schmid und Dralle einen Überblick zum multimodalen Behandlungskonzept beim anaplastischen Schilddrüsenkarzinom (ATC), das eine seltene, aber die bei weitem aggressivste Form dieser Erkrankung darstellt. Molekulargenetische Analysen großer Serien anaplastischer Schilddrüsenkarzinome haben in den letzten Jahren wesentlich zur Charakterisierung der molekularen Pathogenese der ATC beigetragen und molekulare Subtypen identifiziert. Die Genotypisierung der ATC mit der Charakterisierung von Treibermutationen erlaubte die Evaluation einer Reihe von molekular gezielten Therapien. Hierzu zählen insbesondere BRAF-Inhibito- ren und Multityrosinkinaseinhibitoren. Basierend auf dem proimmunogenen Microenvironment des ATC, das durch Kinaseinhibitoren noch verstärkt werden kann, gibt es auch schon erste vielversprechende Daten zur Immuntherapie. Unabhängig davon, dass beim ATC eine - soweit möglich - initiale, aggressive multimodale Therapie mit Chirurgie und Radiochemotherapie einen hohen Stellenwert hat, eröffnen sich hieraus vielversprechende Perspektiven für eine effektivere multimodale Therapie. Die Integration der molekular gezielten Therapie und Immuntherapie in die multidisziplinäre Behandlung von ATCPatienten könnte daher dazu beitragen, die immer noch schlechte Prognose dieser Patienten, v. a. im lokal fortgeschrittenen und/oder metastasierten Stadium, deutlich zu verbessern, Die hierfür in Frage kommenden Patienten sollten daher in national und international koordinierten Multizenterstudien, welche dieses Konzept verfolgen, eingebracht und behandelt werden.

Im zweiten Beitrag fassen Frank-Raue und Raue den aktuellen Wissensstand zum medullären Schilddrüsenkarzinom zusammen. Mutationen des RETProtoonkongens stehen bei diesem Tumortyp nicht nur bei den familiären Formen im Rahmen der multiplen endokrinen Neoplasie Typ 2, sondern auch bei der Mehrzahl der sporadischen medullären Schilddrüsenkarzinome pathogenetisch im Vordergrund. Sie sind auch die Grundlage für die Entwicklung molekular gezielter Therapien für diesen
Tumortyp. Selektive RET-Inhibitoren werden in aktuell laufenden Phase-I/-IIStudien mit äußerst vielversprechenden vorläufigen Ergebnissen untersucht. Darüber hinaus werden in diesem Beitrag aktuelle, angesichts der hohen Prävalenz von Schilddrüsenknoten in Deutschland klinisch äußerst relevante Aspekte zum Calcitonin-Screening erläutert und die Standards für Therapie und strukturierte Nachsorge zusammengefasst.

Der Beitrag von Schmid widmet sich ausführlich den speziellen Diagnosekriterien der "Nichtinvasiven follikulären Neoplasie mit PTC-äquivalenten Kernmerkmalen (NIFTP)“. Darüber hinaus werden weitere Kandidaten für die Einstufung als Neoplasien der Schilddrüse mit unsicherem/geringem Malignitätspotenzial herausgearbeitet, um bei Tumoren mit indolentem klinischem Verlauf eine verstärkt risikoadaptierte Therapie zu erreichen. Damit soll eine Übertherapie vermieden und den Patienten die belastende Krebsdiagnose erspart werden.

Die Konsequenzen, die sich durch die Einführung der NIFTP für die Beurteilung und Aussagekraft der Feinnadelbiopsie ergeben, werden ausführlich und praxisorientiert im darauffolgenden Beitrag von Tötsch und Schmid erörtert.

Im Beitrag von Staubitz und Musholt wird auf Basis der Leitlinien der Chirurgischen Arbeitsgemeinschaft Endokrinologie und der aktuellen internationalen Leitlinien der American Thyroid Association eine kritische Diskussion zum Motto „Weniger ist mehr“ geführt. Dabei 
Hier steht eine Anzeige.

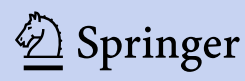


werden auch aktuelle Diskussionen zum Thema Active Surveillance, ein Vorgehen, welches beim papillären Mikrokarzinom, international als Alternative zum direkten chirurgischen Vorgehen zunehmend propagiert wird, mit aufgenommen. Auch äußerst praxisrelevante chirurgische Aspekte, die sich durch die Einführung NIFTP in der aktuellen WHOKlassifikation (4. Aufl.) der Schilddrüsentumoren ergeben, werden ausführlich besprochen.

Im letzten Beitrag geben Spitzweg et al. einen Überblick über medikamentöse Therapieoptionen beim Radiojodrefraktären differenzierten Schilddrüsenkarzinom. Neben den beiden zugelassenen Tyrosinkinaseinhibitoren, Lenvatinib und Sorafenib, stehen eine Reihe weiterer Multityrosinkinaseinhibitoren sowie selektive Inhibitoren im Rahmen von Studien oder im individuellen Heilversuch zur Verfügung. Von großem Potenzial ist auch die Möglichkeit der Reetablierung der Radiojodtherapie durch Reinduktion einer Radiojodaufnahme in den Tumor durch BRAF-Inhibition und/oder MEK-Inhibition, was derzeit in mehreren klinischen Studien untersucht wird. Um die molekular gezielten Therapiemöglichkeiten im Rahmen eines individualisierten Therapiekonzepts voll ausschöpfen zu können, wird heute beim Radiojod-refraktären differenzierten Schilddrüsenkarzinom eine frühzeitige molekulargenetische Analyse des Tumorgewebes sowie die Anbindung an ein spezialisiertes Zentrum empfohlen.

Wir danken allen Autoren für ihre Mühe und Expertise, mit der es ihnen gelungen ist ein eine breitgefächerte und aktuelle Übersicht neuer Aspekte zur Diagnostik und Therapie von Schilddrüsenkarzinomen zusammenzustellen und damit eine sehr erfolgreiche multidisziplinäre Weiterentwicklung aufzuzeigen. Wir sind sicher, dass dies auf ein großes Interesse unserer Leser stoßen wird.

Für die Schriftleiter Christine Spitzweg

Für die Herausgeber

Peter M. Schlag

\section{Korrespondenzadresse}

\section{Prof. Dr. Christine Spitzweg}

Medizinische Klinik und Poliklinik IV, Interdisziplinäres Schilddrüsenzentrum am Klinikum der Universität München (ISKUM), Klinikum der Universität München, LMU München

Marchioninistraße 15, 81377 München, Deutschland

Christine.Spitzweg@med.uni-muenchen.de

Interessenkonflikt. C. Spitzweg erhielt Vortragshonorare von Eisai und Ipsen sowie Honorare für Beratertätigkeit von Eisai. P.M. Schlag gibt an, dass kein Interessenkonflikt besteht.

\section{Neuroonkologie}

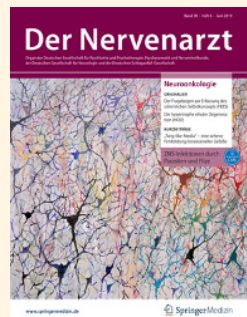

Im Zeitalter der Personalisierten Medizin hat sich die Neuroonkologie grundsätzlich gewandelt. Aus den mannigfaltigen klinischen Gebieten, mit denen sie sich an der Schnittstelle der unterschiedlichsten Disziplinen befasst, wurden für Der Nervenarzt 06/2019 ganz bewusst Themen ausgewählt, die sonst eher selten im Vordergrund stehen.

Schärfen Sie Ihren klinisch-neurologischen Blick und richten sie ihn auf die Bedeutung differentialdiagnostischer Überlegungen und auf das Erkennen und Behandeln neurologischer Symptome und Syndrome (neuro)onkologischer Patienten.

- Hypophysenadenome

- Vestibularisschwannome

- Therapieassoziierte neurologische Symptome onkologischer Patienten

- Herausforderungen und Zukunftsperspektiven in der Neuroonkologie

Suchen Sie noch mehr zum Thema? Mit e.Med - den maßgeschneiderten Fortbildungsabos von Springer Medizin - haben Sie Zugriff auf alle Inhalte von SpringerMedizin.de. Sie können schnell und komfortabel in den für Sie relevanten Zeitschriften recherchieren und auf alle Inhalte im Volltext zugreifen.

Weitere Infos zu e.Med finden Sie auf springermedizin.de unter "Abos" 